\title{
Microscopic calculation of noise current operators for electromagnetic field quantization in absorbing material systems
}

\author{
Omar Di Stefano, Salvatore Savasta and Raffaello Girlanda \\ INFM and Dipartimento di Fisica della Materia e Tecnologie Fisiche Avanzate, \\ Università di Messina, Salita Sperone 31, I-98166 Messina, Italy \\ E-mail: raffaello.girlanda@unime.it
}

\begin{abstract}
We present a microscopic quantization scheme for the electromagnetic field in dispersive and lossy dielectrics of arbitrary geometry. This method also describes anisotropic media and media driven by light field via a spatially nonlocal permittivity. The method removes the need for complicated diagonalization of material, reservoir and field variables and allows us to include the effect of all the material excitations. Dissipation inside the medium is described by considering the coupling of the polarization quanta of the system with the reservoir oscillators in the usual Langevin approach.
\end{abstract}

Keywords: Quantum optics, quantum noise

\section{Introduction}

To describe the quantum features of the propagation of light through dielectric matter, such as optical fibres, dielectric multislab devices, or ultrathin semiconductor resonant layers, quantization of the light field is required. Dispersion in dielectric systems is always associated to loss in accordance with the Kramers-Krönig relations. However, the quantization of the electromagnetic field in absorptive dielectrics is considerably more complicated since the loss couples the field to a reservoir, whose oscillators act as noise sources. A microscopic consistent description of quantized radiation in dispersive and absorptive linear bulk dielectrics was first developed by Huttner and Barnett [1]. They described the dielectric as a polarization field interacting with both the light field and a continuum of reservoir fields, determining absorption, and they diagonalized the coupled radiationmatter Hamiltonian by a generalized Hopfield transformation. This method has been used to calculate the vacuum field fluctuations in absorbing dielectrics [2], and hence to obtain the spontaneous emission rate for an excited atom. The method has been extended to an arbitrary dielectric with a given local complex permittivity [3]. Another approach to the problem of quantization in lossy dielectrics uses Langevin forces to describe noise, and has been applied to the calculation of quantum optical processes in dielectric slabs with local susceptibility $[4,5]$ and to the calculation of Casimir forces in absorbing dielectrics [6,7]. A comprehensive treatment of electromagnetic fields in dispersive and absorbing dielectrics based on the Langevin forces has been given in $[8,9]$. The method has also been applied to the analysis of propagation of nonclassical light pulses in slabs [10,11]. A three-dimensional (3D) quantization scheme for homogeneous absorbing dielectrics based on the Langevin forces and the Green function method has been presented in [12]. Ho Trung Dung et al [13] and Scheel et al [14] have extended the quantization scheme in inhomogeneous media to include the full 3D nature of the electromagnetic field starting from the phenomenological Maxwell equations. In particular, Scheel et al have proved that the fundamental equal-time commutation relations of QED are preserved for an arbitrary space-dependent, Kramers-Krönig consistent permittivity. A $3 \mathrm{D}$ quantization scheme taking explicitly into account the finite extent of media has been recently presented by us $[15,19]$.

A different general 3D quantization scheme that makes use of a set of auxiliary fields, followed by a canonical quantization 
procedure has been developed by Tip [16]. Recently, the equivalence of the quantization schemes by Scheel et al [14] and by Tip [16] has been demonstrated [17].

Microscopic methods require complicated diagonalization procedures of material, reservoir and field variables. The Langevin method has the advantage that it is valid for an arbitrary dielectric function and that removes the need for complicated diagonalization procedures. However, this method is to some degree phenomenological as it is not based on a microscopic model for the dielectric material. As a consequence the commutation relations for the noise currents are determined $a$ posteriori, by imposing that the obtained quantized fields obey the canonical commutation relations. Gruner and Welsch, reinserting the Huttner and Barnett solution for the field quantization in an infinite bulk medium into the Maxwell equations, provided a microscopic derivation of the noise current operators [18]. By this procedure one has to solve the quantization problem before calculating the noise operators.

Here we consider the most general non-magnetic linear scattering system that can be described by a causal nonlocal susceptibility tensor $\chi_{i, j}\left(\boldsymbol{r}, \boldsymbol{r}^{\prime}, \omega\right)$ (of course for systems or part of a system that can be described by a local susceptibility, $\chi_{i j}\left(\boldsymbol{r}, \boldsymbol{r}^{\prime}\right)$ reduces to $\left.\chi_{i j}(\boldsymbol{r}) \delta\left(\boldsymbol{r}-\boldsymbol{r}^{\prime}\right)\right)$ [19]. Electronic states of semiconductors and semiconductor quantum structures, and also all those systems whose susceptibility depends on wavevectors are examples of systems driven via a nonlocal susceptibility. We present a microscopic quantization scheme which avoids diagonalization procedures. Our microscopic method is analogous to the method based on Langevin forces applied to field quantization in media with local susceptibilities [8]. This scheme simply provides a microscopic direct derivation of the general dielectric function and of the Langevin forces and naturally links the field quantization in absorbing dielectrics with the standard Langevin approach adopted to include dissipation in the quantum dynamics of a harmonic oscillator [20]. Moreover, this approach extends previous treatments by allowing us to treat anisotropic media and media driven by the light field via a spatially nonlocal permittivity.

\section{The photon-electron interaction Hamiltonian}

In order to describe the interaction of light with matter inside the dielectric medium, we will adopt the Power-ZienauWolley (PZW) or multipolar interaction Hamiltonian [21] in the dipole approximation.

The canonical coordinate for the electromagnetic field is the transverse vector potential operator $\hat{A}(r)$. Its conjugate momentum in the PZW gauge is given by $\hat{\Pi}=-\hat{\boldsymbol{D}}(\boldsymbol{r}) / \epsilon_{0}$, where $\hat{D}$ is the transverse displacement operator. We point out that $\hat{A}$ and $\hat{D}$ commute with all the operators of the material system (we refer to equal-time commutators). In order to apply the dipole approximation to an extended system, we must divide the whole medium into many cells of the same volume $\Omega$, and each cell is specified by a position vector $r$. These cells must have a much smaller size than the wavelength of the electric field in consideration (a possible choice is to make the crystal unit cell such a unit). The PZW Hamiltonian, in the extended dipole approximation, can be written as

$$
\hat{H}_{I}=-\frac{\Omega}{\epsilon_{0}} \sum_{r} \hat{\boldsymbol{D}}(\boldsymbol{r}) \cdot \hat{\boldsymbol{P}}(\boldsymbol{r})+\frac{\Omega}{2 \epsilon_{0}} \sum_{r} \hat{\boldsymbol{P}}^{2}(\boldsymbol{r}),
$$

where $\hat{\boldsymbol{P}}=\hat{\boldsymbol{P}}^{\mathrm{tr}}(\boldsymbol{r})+\hat{\boldsymbol{P}}^{\mathrm{L}}(\boldsymbol{r})$ is the polarization operator of the material system, with $\hat{\boldsymbol{P}}^{\mathrm{tr}}(\boldsymbol{r})$ and $\hat{\boldsymbol{P}}^{\mathrm{L}}(\boldsymbol{r})$ respectively being the transverse and the longitudinal polarization-density operators. The terms $\hat{\boldsymbol{D}}(\boldsymbol{r}) \cdot \hat{\boldsymbol{P}}(\boldsymbol{r})$ and $\hat{\boldsymbol{P}}(\boldsymbol{r}) \cdot \hat{\boldsymbol{P}}^{\mathrm{tr}}(\boldsymbol{r})$ describe the interaction of the medium with the transverse electromagnetic field, while the term $\hat{\boldsymbol{P}}(\boldsymbol{r}) \cdot \hat{\boldsymbol{P}}^{\mathrm{L}}(\boldsymbol{r})$ describes the energy of the longitudinal field $\hat{\boldsymbol{E}}^{\mathrm{L}}(\boldsymbol{r})=-\hat{\boldsymbol{P}}^{\mathrm{L}}(\boldsymbol{r}) / \varepsilon_{0}$. Although the results presented here can be applied to any material excitation interacting with the electric field, such as optical phonons, plasmons and excitons, in the following we specialize to excitations arising from electronic interband transitions.

The electronic polarization-density operator can be expressed as $\boldsymbol{P}(\boldsymbol{r})=\sum_{j} \boldsymbol{P}_{\boldsymbol{r}}\left(\boldsymbol{x}_{j}\right)$, with the contribution of the $j$ th electron given by

$$
\boldsymbol{P}_{r}\left(\boldsymbol{x}_{j}\right)=-\frac{1}{\Omega} \int_{\Omega_{r}} \mathrm{~d} \boldsymbol{x}^{\prime} \delta\left(\boldsymbol{x}_{j}-\boldsymbol{r}-\boldsymbol{x}^{\prime}\right) e \boldsymbol{x}^{\prime},
$$

with integration limited to the volume of the cell, $\Omega$ being the cell volume. The polarization density operator in second quantization form is given by

$$
\hat{\boldsymbol{P}}(\boldsymbol{r})=\int_{-\infty}^{\infty} \mathrm{d} \boldsymbol{x} \hat{\Psi}^{\dagger}(\boldsymbol{x}) \boldsymbol{P}_{\boldsymbol{r}}(\boldsymbol{x}) \hat{\Psi}(\boldsymbol{x}),
$$

where $\hat{\Psi}$ and $\hat{\Psi}^{\dagger}$ are fermion field operators. We expand the field operators in terms of the wavefunctions of conduction and valence bands

$$
\hat{\Psi}(\boldsymbol{x})=\sum_{\lambda_{\mathrm{v}}} \psi_{\lambda_{\mathrm{v}}}(\boldsymbol{x}) \hat{v}_{\lambda_{\mathrm{v}}}+\sum_{\lambda_{\mathrm{c}}} \psi_{\lambda_{\mathrm{c}}}(\boldsymbol{x}) \hat{c}_{\lambda_{\mathrm{c}}} .
$$

We have considered a two-band direct-gap model where $v(c)$ labels the valence (conduction) band. In equation (3) $\hat{c}_{\lambda_{\mathrm{c}}}$ $\left(\hat{v}_{\lambda_{v}}\right)$ are annihilation operators of electrons in the conduction subband $\lambda_{\mathrm{c}}$ (in the valence subband $\lambda_{\mathrm{V}}$ ). The one-particle valence and conduction wavefunctions on the right-hand side of (3), in the envelope function approximation [22], are given by

$$
\begin{aligned}
& \psi_{\lambda_{\mathrm{v}}}(\boldsymbol{x})=u_{\lambda_{\mathrm{v}}}(\boldsymbol{x}) \zeta_{\lambda_{\mathrm{v}}}(\boldsymbol{x}), \\
& \psi_{\lambda_{\mathrm{c}}}(\boldsymbol{x})=u_{\lambda_{\mathrm{c}}}(\boldsymbol{x}) \zeta_{\lambda_{\mathrm{c}}}(\boldsymbol{x}),
\end{aligned}
$$

where $\boldsymbol{x} \equiv\left(x_{1}, x_{2}, x_{3}\right)$ labels the electron coordinates and $\lambda_{\mathrm{c}}$ and $\lambda_{\mathrm{v}}$ are collective quantum numbers representing the set of quantum number labelling the electronic energy levels of the system, including eventually the $1 \mathrm{D}, 2 \mathrm{D}$ or $3 \mathrm{D}$ wavevector according to the translational properties of the system. In equations (4) and (5) $u_{\lambda_{\mathrm{v}}\left(\lambda_{\mathrm{c}}\right)}(\boldsymbol{x})$ is the periodic part of the Bloch functions normalized inside the unit cell. The envelope functions $\zeta_{\lambda_{v}\left(\lambda_{c}\right)}(x)$ are slowly varying functions with respect to the dimensions of the cells and can be considered as constant inside each elementary cell. They are normalized as follows:

$$
\sum_{r} \zeta_{\lambda_{\mathrm{v}}\left(\lambda_{\mathrm{c}}\right)}^{*}(\boldsymbol{r}) \zeta_{\lambda_{\mathrm{v}}\left(\lambda_{\mathrm{c}}\right)}(\boldsymbol{r})=1
$$

These envelope functions describe the propagation and the confinement of the electrons in a material systems with eventually broken translation symmetry. 

that

By considering only interband transitions, thus assuming

$$
\int_{-\infty}^{\infty} \mathrm{d} \boldsymbol{x} \psi_{\lambda_{\mathrm{v}}\left(\lambda_{\mathrm{c}}\right)}^{\star}(\boldsymbol{x}) \boldsymbol{P}_{\boldsymbol{r}}(\boldsymbol{x}) \psi_{\lambda_{\mathrm{v}}\left(\lambda_{\mathrm{c}}\right)}(\boldsymbol{x})=0,
$$

and introducing the field operators (3) into (2), we obtain

$$
\hat{\boldsymbol{P}}(\boldsymbol{r})=\sum_{\lambda_{\mathrm{c}}, \lambda_{\mathrm{v}}} c_{\lambda_{\mathrm{c}}}^{\dagger} v_{\lambda_{\mathrm{v}}} \int_{-\infty}^{\infty} \mathrm{d} \boldsymbol{x} \psi_{\lambda_{\mathrm{c}}}^{\star}(\boldsymbol{x}) \boldsymbol{P}_{\boldsymbol{r}}(\boldsymbol{x}) \psi_{\lambda_{\mathrm{v}}}(\boldsymbol{x})+\text { H.c. }
$$

We introduce in equation (8) the explicit electronic wavefunctions given in (4) and (5). We then write the integral in (8) as a sum of integrals limited to the volume of each cell. We can thus take advantage of the slowly varying character of the function $\zeta_{\lambda_{v}\left(\lambda_{c}\right)}(x)$ considering this envelope function as a constant $\zeta_{\lambda_{\mathrm{v}}\left(\lambda_{\mathrm{c}}\right)}(\boldsymbol{r})$ inside each cell. We obtain

$$
\hat{\boldsymbol{P}}(\boldsymbol{r})=\sum_{\beta} \boldsymbol{\mu}_{\beta} \rho_{\beta}(\boldsymbol{r}) \hat{c}_{\lambda_{\mathrm{c}}}^{\dagger} \hat{v}_{\lambda_{v}}+\text { H.c. },
$$

where

$$
\rho_{\beta}(\boldsymbol{r})=\tilde{\zeta}_{\lambda_{\mathrm{c}}}^{*}(\boldsymbol{r}) \tilde{\zeta}_{\lambda_{\mathrm{v}}}(\boldsymbol{r})
$$

where $\beta \equiv\left(\lambda_{\mathrm{c}}, \lambda_{\mathrm{v}}\right)$ and $\tilde{\zeta}=\zeta / \sqrt{\Omega} . \quad \boldsymbol{\mu}_{\beta}$ is the interbanddipole matrix element given by

$$
\boldsymbol{\mu}_{\beta}=-\int_{\Omega_{j}} \mathrm{~d} \boldsymbol{x} u_{\lambda_{\mathrm{c}}}^{*}(\boldsymbol{x}) e \boldsymbol{x} u_{\lambda_{\mathrm{v}}}(\boldsymbol{x}) .
$$

If the medium is isotropic the interband-dipole matrix is simply given by a scalar $\left(\left(\mu_{\beta}\right)_{i}=\mu_{\beta}\right)$. By replacing the valence band operators $v_{\lambda_{\mathrm{v}}}$ with hole operators $h_{\lambda_{\mathrm{v}}}^{\dagger}, \hat{\boldsymbol{P}}(\boldsymbol{r})$ can be written as

$$
\hat{\boldsymbol{P}}(\boldsymbol{r})=\sum_{\beta} \boldsymbol{\mu}_{\beta} \hat{B}_{\beta} \rho_{\beta}^{*}(\boldsymbol{r})+\text { H.c. }=\hat{\boldsymbol{P}}^{+}(\boldsymbol{r})+\hat{\boldsymbol{P}}^{-}(\boldsymbol{r})
$$

where we have defined the excitation operator as

$$
\hat{B}_{\beta}=c_{\lambda_{\mathrm{c}}} h_{\lambda_{\mathrm{v}}},
$$

and

$$
\hat{\boldsymbol{P}}^{+}(\boldsymbol{r})=\left[\hat{\boldsymbol{P}}^{-}(\boldsymbol{r})\right]^{\dagger}=\sum_{\beta} \hat{\boldsymbol{P}}_{\beta}^{+}(\boldsymbol{r}),
$$

with

$$
\hat{\boldsymbol{P}}_{\beta}^{+}(\boldsymbol{r})=\boldsymbol{\mu}_{\beta} \hat{B}_{\beta} \rho_{\beta}^{*}(\boldsymbol{r}) .
$$

We have now determined the second quantization form of the polarization in terms of the excitations of the material system, and hence we have explicitly determined the second quantization form of $H_{I}$.

As the wavelength of the field at optical frequencies is much larger than the dimensions of the unit cell, we can perform the continuum limit. The second quantization form of (1) can be thus written as

$$
\hat{H}_{I}=-\frac{1}{\epsilon_{0}} \int \mathrm{d}^{3} r \hat{\boldsymbol{D}}(\boldsymbol{r}) \cdot \hat{\boldsymbol{P}}(\boldsymbol{r})+\frac{1}{2 \epsilon_{0}} \int \mathrm{d}^{3} r \hat{\boldsymbol{P}}^{2}(\boldsymbol{r})
$$

where integration is extended to the whole volume of the system. Analogously to the total polarization operator, the electric field operator can also be separated into the positive and negative frequency components in the usual way, $\hat{\boldsymbol{E}}(\boldsymbol{r}, t)=$
$\hat{\boldsymbol{E}}^{+}(\boldsymbol{r}, t)+\hat{\boldsymbol{E}}^{-}(\boldsymbol{r}, t)$ [20]. For future use it is useful to introduce the time Fourier transforms

$$
\begin{aligned}
& \hat{\boldsymbol{E}}^{+}(\boldsymbol{r}, t)=\int_{0}^{\infty} \mathrm{d} \omega \mathrm{e}^{-\mathrm{i} \omega t} \hat{\boldsymbol{E}}^{+}(\boldsymbol{r}, \omega), \\
& \hat{\boldsymbol{P}}^{+}(\boldsymbol{r}, t)=\int_{0}^{\infty} \mathrm{d} \omega \mathrm{e}^{-\mathrm{i} \omega t} \hat{\boldsymbol{P}}^{+}(\boldsymbol{r}, \omega) .
\end{aligned}
$$

The negative frequency components are given by the Hermitian conjugate of the positive frequency operators [8]. In the usual rotating wave approximation [20], equation (15) becomes

$$
\begin{aligned}
\hat{H}_{I} & =\left(-\frac{1}{\epsilon_{0}} \int \mathrm{d}^{3} r \hat{\boldsymbol{D}}^{+}(\boldsymbol{r}) \cdot \hat{\boldsymbol{P}}^{-}(\boldsymbol{r})\right. \\
& \left.+\frac{1}{2 \epsilon_{0}} \int \mathrm{d}^{3} r \hat{\boldsymbol{P}}^{+}(\boldsymbol{r}) \hat{\boldsymbol{P}}^{-}(\boldsymbol{r})\right)+ \text { H.c. }
\end{aligned}
$$

\section{Heisenberg-Langevin equations for the material system}

When analysing the linear response, i.e. when considering a regime of low optical excitation, the excitation operators can be assumed to be Boson operators. In the absence of interaction with light, the Hamiltonian describing the electronic excitation of the medium can be written simply in terms of Boson excitation operators,

$$
\hat{H}_{m}=\sum_{\beta} \hbar \omega_{\beta} \hat{B}_{\beta}^{\dagger} \hat{B}_{\beta}
$$

where $\hbar \omega_{\beta}$ are the energy levels of the system. The total Hamiltonian acting on the material system is given by

$$
\hat{H}_{\text {tot }}=\hat{H}_{m}+\hat{H}_{I}
$$

By using the rotating wave approximation (16) for $H_{I}$, the Heisenberg equations of motion for the excitation operators,

$$
\mathrm{i} \frac{\partial}{\partial t} \hat{B}_{\beta}=\frac{1}{\hbar}\left[\hat{B}_{\beta}, \hat{H}_{\mathrm{tot}}\right]
$$

are given by

$$
\mathrm{i} \frac{\partial}{\partial t} \hat{B}_{\beta}=\omega_{\beta} \hat{B}_{\beta}-\frac{\left(\mu_{\beta}\right)_{l}}{\hbar} \int_{-\infty}^{\infty} \mathrm{d} \boldsymbol{x}^{\prime} \rho_{\beta}\left(\boldsymbol{x}^{\prime}\right) \hat{E}_{l}^{+}\left(\boldsymbol{x}^{\prime}, t\right),
$$

where we have used the relation

$$
\hat{\boldsymbol{E}}^{ \pm}(\boldsymbol{r}, t)=\frac{1}{\epsilon_{0}}\left(\hat{\boldsymbol{D}}^{ \pm}(\boldsymbol{r}, t)-\hat{\boldsymbol{P}}^{ \pm}(\boldsymbol{r}, t)\right) .
$$

From equation (20) we can directly derive a set of equations for the Fourier components $\hat{P}^{+}(r, \omega)$,

$$
\begin{gathered}
\left(\omega-\omega_{\beta}\right) \hat{P}_{\beta, m}^{+}(\boldsymbol{r}, \omega)=-\frac{\left(\mu_{\beta}\right)_{m}\left(\mu_{\beta}\right)_{l}}{\hbar} \rho_{\beta}^{\star}(\boldsymbol{r}) \\
\times \int_{-\infty}^{\infty} \mathrm{d} \boldsymbol{x}^{\prime} \rho_{\beta}\left(\boldsymbol{x}^{\prime}\right) \hat{E}_{l}^{+}\left(\boldsymbol{x}^{\prime}, \omega\right) .
\end{gathered}
$$

We now introduce the effect of dissipation due to the degrees of freedom internal to the material system. We introduce a set of harmonic oscillators interacting with the excitation operators. Following the usual Langevin approach, the resulting Heisenberg-Langevin equations for the electronic excitations can be derived from equation (20) by including 
a damping term $-\mathrm{i} \gamma_{\beta} \hat{B}_{\beta}$ and a quantum noise Langevin operator $-\mathrm{i} \sqrt{2 \gamma_{\beta}} \hat{F}_{\beta}(t)$ [20]. The noise Langevin operator, $\hat{F}_{\beta}(t)$, has zero expectation values and satisfies the following commutation relations:

$$
\begin{gathered}
{\left[\hat{F}_{\beta}(t), \hat{F}_{\beta^{\prime}}^{\dagger}\left(t^{\prime}\right)\right]=\delta_{\beta, \beta^{\prime}} \delta\left(t-t^{\prime}\right),} \\
{\left[\hat{F}_{\beta}(t), \hat{F}_{\beta^{\prime}}\left(t^{\prime}\right)\right]=0 .}
\end{gathered}
$$

For a zero-temperature reservoir, force-force correlations are given by

$$
\left\langle\hat{F}_{\beta}(t) \hat{F}_{\beta^{\prime}}^{\dagger}\left(t^{\prime}\right)\right\rangle=\delta_{\beta, \beta^{\prime}} \delta\left(t-t^{\prime}\right),
$$

and

$$
\left\langle\hat{F}_{\beta}(t) \hat{F}_{\beta^{\prime}}\left(t^{\prime}\right)\right\rangle=\left\langle\hat{F}_{\beta}^{\dagger}(t) \hat{F}_{\beta^{\prime}}\left(t^{\prime}\right)\right\rangle=0 .
$$

Introducing the Fourier transform for the noise operator,

$$
\hat{F}_{\beta}(t)=\frac{1}{\sqrt{2 \pi}} \int_{0}^{\infty} \mathrm{d} \omega \mathrm{e}^{-\mathrm{i} \omega t} \hat{F}_{\beta}(\omega)
$$

into equation (22) we obtain

$$
\left[\hat{F}_{\beta}(\omega), \hat{F}_{\beta^{\prime}}^{\dagger}\left(\omega^{\prime}\right)\right]=\delta_{\beta, \beta^{\prime}} \delta\left(\omega-\omega^{\prime}\right) .
$$

Introducing the effects of the interaction with the reservoir into equation (21), we obtain the Heisenberg-Langevin equations of motion for the polarization operators

$$
\begin{aligned}
(\omega- & \left.\omega_{\beta}+\mathrm{i} \gamma_{\beta}\right) \hat{P}_{\beta, m}^{+}(\boldsymbol{r}, \omega)=-\frac{\left(\mu_{\beta}\right)_{m}\left(\mu_{\beta}\right)_{l}}{\hbar} \rho_{\beta}^{\star}(\boldsymbol{r}) \\
& \times \int_{-\infty}^{\infty} \mathrm{d} \boldsymbol{x} \rho_{\beta}\left(\boldsymbol{x}^{\prime}\right) \hat{E}_{l}^{+}\left(\boldsymbol{x}^{\prime}, \omega\right) \\
& -\mathrm{i}\left(\mu_{\beta}\right)_{m} \sqrt{\frac{\gamma_{\beta}}{\pi}} \hat{F}_{\beta^{\prime}}(\omega) \rho_{\beta}^{*}\left(\boldsymbol{x}^{\prime}\right) .
\end{aligned}
$$

Inverting equation (24) and summing over the e-h levels $\beta$ we obtain an expression for the total positive component of the electronic polarization,

$$
\begin{gathered}
\hat{P}_{m}^{+}(\boldsymbol{r}, \omega)=\epsilon_{0} \int_{-\infty}^{\infty} \mathrm{d} \boldsymbol{r}^{\prime} \chi_{m, l}\left(\boldsymbol{r}, \boldsymbol{r}^{\prime}, \omega\right) \hat{E}_{l}^{+}\left(\boldsymbol{r}^{\prime}, \omega\right) \\
+\sqrt{\frac{\hbar}{\pi}} \hat{f}_{m}(\boldsymbol{r}, \omega),
\end{gathered}
$$

where the linear nonlocal susceptibility is given by

$$
\chi_{m, l}\left(\boldsymbol{r}, \boldsymbol{r}^{\prime}, \omega\right)=\sum_{\beta}\left(\tilde{\chi}_{\beta}\right)_{m, l} \rho_{\beta}^{*}(\boldsymbol{r}) \rho_{\beta}\left(\boldsymbol{r}^{\prime}\right),
$$

with

$$
\left(\tilde{\chi}_{\beta}\right)_{m, l}=-\frac{1}{\epsilon_{0} \hbar} \frac{\left(\mu_{\beta}\right)_{m}\left(\mu_{\beta}\right)_{l}}{\omega-\omega_{\beta}+\mathrm{i} \gamma_{\beta}},
$$

and where the noise operator is given by

$$
\hat{f}_{m}(\boldsymbol{r}, \omega)=\sum_{\beta} \xi_{\beta, m}(\boldsymbol{r}, \omega) \hat{F}_{\beta}(\omega),
$$

with

$$
\xi_{\beta, m}(\boldsymbol{r}, \omega)=-\mathrm{i} \sqrt{\frac{\gamma_{\beta}}{\hbar}} \frac{\left(\mu_{\beta}\right)_{m}}{\omega-\omega_{\beta}+\mathrm{i} \gamma_{\beta}} \rho_{\beta}^{*}(\boldsymbol{r}) .
$$

The quantum noise operators $\hat{f}_{m}(\boldsymbol{r}, \omega)$, defined in equation (28), obeys the following commutation relations:

$$
\begin{gathered}
{\left[\hat{f}_{l}(\boldsymbol{r}, \omega), \hat{f}_{m}^{\dagger}\left(\boldsymbol{r}^{\prime}, \omega^{\prime}\right)\right]=\epsilon_{0} \chi_{l m}^{\Im}\left(\boldsymbol{r}, \boldsymbol{r}^{\prime}, \omega\right) \delta\left(\omega-\omega^{\prime}\right) .} \\
{\left[\hat{f}_{l}(\boldsymbol{r}, \omega), \hat{f}_{m}\left(\boldsymbol{r}^{\prime}, \omega^{\prime}\right)\right]=0 .}
\end{gathered}
$$

The relations that we have obtained in this section can be applied to specific cases. If we consider an anisotropic system driven by the local susceptibility

$$
\chi_{l m}^{I}\left(\boldsymbol{r}, \boldsymbol{r}^{\prime}, \omega\right)=\chi_{l m}^{I}(\boldsymbol{r}, \omega) \delta\left(\boldsymbol{r}-\boldsymbol{r}^{\prime}\right)
$$

equation (29) becomes

$$
\left[\hat{f}_{l}(\boldsymbol{r}, \omega), \hat{f}_{m}^{\dagger}\left(\boldsymbol{r}^{\prime}, \omega^{\prime}\right)\right]=\epsilon_{0} \chi_{l m}^{I}(\boldsymbol{r}, \omega) \delta\left(\boldsymbol{r}-\boldsymbol{r}^{\prime}\right) \delta\left(\omega-\omega^{\prime}\right) .
$$

The susceptibility of an isotropic local system is

$$
\chi_{l m}^{I}\left(\boldsymbol{r}, \boldsymbol{r}^{\prime}, \omega\right)=\chi^{I}(\boldsymbol{r}, \omega) \delta_{l m} \delta\left(\boldsymbol{r}-\boldsymbol{r}^{\prime}\right),
$$

and we obtain

$$
\left[\hat{f}_{l}(\boldsymbol{r}, \omega), \hat{f}_{m}^{\dagger}\left(\boldsymbol{r}^{\prime}, \omega^{\prime}\right)\right]=\chi^{I}(\boldsymbol{r}, \omega) \delta_{l m} \delta\left(\boldsymbol{r}-\boldsymbol{r}^{\prime}\right) \delta\left(\omega-\omega^{\prime}\right) .
$$

\section{A Fredholm equation for the light field}

The total Hamiltonian acting on the electromagnetic field is given by

$$
H=H_{\mathrm{ph}}+H_{I} \text {. }
$$

$H_{\mathrm{ph}}$ is the photon Hamiltonian in the absence of the material system. In the multipolar form of the interaction, adopted here, the field coordinate is given by the vector potential, while the conjugate momentum is given by the displacement operator. From the Heisenberg equations of motions for the field variables we can obtain an operator wave equation for the electric field operator $\hat{\boldsymbol{E}}(\boldsymbol{r}, t)$,

$$
\nabla \times \nabla \times \hat{\boldsymbol{E}}^{+}(\boldsymbol{r}, \omega)-\frac{\omega^{2}}{c^{2}} \hat{\boldsymbol{E}}^{+}(\boldsymbol{r}, \omega)=\frac{\omega^{2}}{\varepsilon_{0} c^{2}} \hat{\boldsymbol{P}}^{+}(\boldsymbol{r}, \omega)
$$

Introducing into equation (33) the expression (25) obtained for the electronic polarization, we obtain in Cartesian components

$$
\begin{aligned}
& {\left[\nabla \times \nabla \times \hat{E}^{+}(\boldsymbol{r}, \omega)\right]_{i}=\frac{\omega^{2}}{c^{2}} \int_{-\infty}^{\infty} \mathrm{d} z^{\prime} \chi_{i j}\left(\boldsymbol{r}, \boldsymbol{r}^{\prime}, \omega\right) \hat{E}_{j}^{+}\left(\boldsymbol{r}^{\prime}, \omega\right)} \\
& \quad+\mathrm{i} \omega \mu_{0} \hat{j}_{i}(\boldsymbol{r}, \omega)
\end{aligned}
$$

where

$$
\hat{j}_{i}(\boldsymbol{r}, \omega)=-\mathrm{i} \omega \sqrt{\frac{\hbar}{\pi}} \hat{f}_{i}(\boldsymbol{r}, \omega) .
$$

Equation (34) is a Fredholm integral equation with a source term. This Fredholm equation, together with the commutation relations of the noise operators,

$$
\begin{gathered}
{\left[\hat{j}_{i}(\boldsymbol{r}, \omega), \hat{j}_{j}^{\dagger}\left(\boldsymbol{r}^{\prime}, \omega^{\prime}\right)\right]=\frac{\hbar}{\pi \mu_{0}} \frac{\omega^{2}}{c^{2}} \chi_{i, j}^{I}\left(\boldsymbol{r}, \boldsymbol{r}^{\prime}, \omega\right) \delta\left(\omega-\omega^{\prime}\right),} \\
{\left[\hat{j}_{i}(\boldsymbol{r}, \omega), \hat{j}_{j}\left(\boldsymbol{r}^{\prime}, \omega^{\prime}\right)\right]=0,}
\end{gathered}
$$

is the starting point for field quantization in systems described by a general $3 \mathrm{D}$ absorbing and dispersive material system which can even be anisotropic and/or driven via a nonlocal susceptibility. 
This quantization scheme, which allowed us to obtain equations (29) and (34), is based on a microscopic linear twoband model. However, since the influence of the medium can be described only in terms of the complex frequency-dependent and nonlocal susceptibility of the medium, equations (34) and (35) establish a quantization scheme which can be applied beyond this model.

\section{Conclusions}

We have presented a microscopic scheme for field quantization in absorbing and dispersive dielectrics. It provides a simple microscopic derivation of the general dielectric function and of the noise currents that act as source terms in the operator wave equation for the electromagnetic field. This approach naturally links the field quantization in absorbing dielectrics with the standard Langevin approach adopted to include dissipation in the quantum dynamics of a harmonic oscillator [20]. Furthermore, this approach produces equations of motion and a dielectric function which are in close analogy with the corresponding quantities obtained in the framework of classical, or semiclassical treatments of linear light-matter interaction, thus providing a direct precise link with the well known classical theories. By this approach we have derived for the first time noise current operators for general anisotropic and/or spatially nonlocal media.

\section{Acknowledgment}

The work was partially supported by the MURST through COFIN'97.

\section{References}

[1] Huttner B and Barnett S M 1992 Phys. Rev. A 464306

[2] Barnett S M, Huttner B and Loudon R 1992 Phys. Rev. Lett. 68 3698

[3] Gruner T and Welsch D G 1996 Phys. Rev. A 531818

[4] Knöll L and Leonhardt U 1992 J. Mod. Opt. 391253

[5] Leonhardt U 1993 J. Mod. Opt. 401123

[6] Kupiszewska D 1992 Phys. Rev. A 462286

[7] Matloob R 1999 Phys. Rev. A 603421

[8] Matloob R, Loudon R, Barnett S M and Jeffers J 1995 Phys. Rev. A $\mathbf{5 2} 4823$

[9] Di Stefano O, Savasta S and Girlanda R 2001 J. Mod. Opt. 48 67-84

[10] Artoni M and Loudon R 1997 Phys. Rev. A 551347

[11] Artoni M and Loudon R 1999 Phys. Rev. A 592279

[12] Matloob R 1999 Phys. Rev. A 6050

[13] Ho Trung Dung, L Knöll and Welsch D G 1998 Phys. Rev. A 573931

[14] Scheel S, Knöll L and Welsch D-G 1998 Phys. Rev. A 58700

[15] Di Stefano O, Savasta S and Girlanda R 2000 Phys. Rev. A 61 023803

[16] Tip A 1998 Phys. Rev. A 6574818

[17] Tip A, Knöll L, Scheel S and Welsch D-G 2001 Phys. Rev. A 63043806

[18] Gruner T and Welsch D G 1995 Phys. Rev. A 513246

[19] Di Stefano O, Savasta S and Girlanda R 1999 Phys. Rev. A 60 1614-25

[20] Mandel L and Wolf E 1995 Optical Coherence and Quantum Optics (Cambridge: Cambridge University Press)

Scully M O and Zubairy M S 1997 Quantum Optics (Cambridge: Cambridge University Press)

[21] Cohen-Tannoudji C, Dupont-Roc J and Grynberg G 1987 Photons et Atomes (InterEditions/Editions du CNRS)

[22] Bastard G, Mendez E E and Esaki L L 1982 Phys. Rev. B 26 1974 